\title{
Incidencia y características clínicas de la enfermedad tiroidea en pacientes con neoplasias malignas tratados con inhibidores de punto de control inmunológico
}

\author{
Dalia Cuenca, ${ }^{1}$ Elina Rodríguez-Meléndez, ${ }^{2}$ Mercedes Aguilar-Soto, ${ }^{1}$ Alain Sánchez-Rodríguez, ${ }^{1}$ \\ Nicole Íñiguez-Ariza, ${ }^{3}$ Guillermo Olivares-Beltrán, ${ }^{4}$ Raquel Gerson-Cwilich ${ }^{4}$ y Moisés Mercado5* \\ ${ }^{1}$ American British Cowdray Medical Center, Departamento de Medicina; ${ }^{2}$ Hospital "Luis Vernaza y Solca", Departamento de Oncología Médica, \\ Guayaquil, Ecuador; ${ }^{3}$ Instituto Nacional de Ciencias Médicas y Nutrición "Salvador Zubirán", Departamento de Endocrinología y Metabolismo; \\ ${ }^{4}$ American British Cowdray Medical Center, Centro de Cáncer; ${ }^{5}$ Instituto Mexicano del Seguro Social, Centro Médico Nacional Siglo XXI, Hospital \\ de Especialidades. ${ }^{1,3-5}$ Ciudad de México, México
}

\section{Resumen}

Introducción: Los inhibidores del punto de control inmunológico (IPCi) son utilizados en los últimos años en el tratamiento de neoplasias malignas avanzadas, con ellos se ha logrado un aumento significativo de la supervivencia; sin embargo, su uso se ha asociado a incremento del riesgo de enfermedades autoinmunes. Objetivo: Describir la incidencia y las características clínicas de los pacientes tratados con IPCi que desarrollaron tiroidopatía. Métodos: Se revisaron retrospectivamente los expedientes de todos los pacientes que recibieron IPCi en los últimos tres años y se identificaron aquellos que desarrollaron anomalías tiroideas. Resultados: La prevalencia de tiroiditis fue de $7 \%$, con una incidencia de $21.4 \%$ pacientes $/$ mes. La mediana del tiempo para el desarrollo de tiroiditis fue de 63 días. La mayoría de los pacientes presentó síntomas leves o moderados y no requirió hospitalización, si bien todos menos uno desarrollaron hipotiroidismo permanente y requirieron terapia de reemplazo hormonal con levotiroxina. Conclusiones: La disfunción tiroidea secundaria a inmunoterapia es una entidad común en nuestra población. El cuadro clínico suele ser leve y no requiere suspender el tratamiento; sin embargo, debido a la alta incidencia de este evento adverso, los médicos no oncólogos deben estar familiarizados con su diagnóstico y tratamiento, para brindar un manejo multidisciplinario.

PALABRAS CLAVE: Inhibidores del punto de control inmunológico. Tiroiditis. Toxicidad. Inmunoterapia. Hipotiroidismo.

\section{Incidence and clinical characteristics of thyroid abnormalities in cancer patients treated with immune checkpoint inhibitors}

\begin{abstract}
Introduction: Immune checkpoint inhibitors (ICI) are a group of drugs that have been used in recent years for the treatment of advanced malignancies such as melanoma, non-small cell lung cancer and other tumors, significantly increasing survival. However, the use of $\mathrm{ICI}$ has been associated with an increased risk of autoimmune diseases, with endocrine organs, specifically the thyroid, being highly susceptible to this phenomenon. Objective: To describe the incidence and clinical characteristics of patients treated with $\mathrm{ICI}$ who develop thyroid disease. Methods: The medical records of all patients who received ICI treatment within the last three years were retrospectively reviewed, with those who developed thyroid abnormalities being identified. Results: The prevalence of thyroiditis was $7 \%$, with an incidence of $21.4 \%$ of patients-month. Median time for the development of thyroiditis was 63 days. Most patients had mild or moderate symptoms and did not require hospitalization, although all but
\end{abstract}

Correspondencia:

*Moisés Mercado

E-mail: moises.mercado@endocrinologia.org.mx
Fecha de recepción: 04-08-2020

Fecha de aceptación: 17-09-2020

DOI: $10.24875 / G M M .20000547$
Gac Med Mex. 2021;157:305-310

Disponible en PubMed

www.gacetamedicademexico.com

0016-3813/@ 2020 Academia Nacional de Medicina de México, A.C. Publicado por Permanyer. Este es un artículo open access bajo la licencia CC BY-NC-ND (http://creativecommons.org/licenses/by-nc-nd/4.0/). 
one developed permanent hypothyroidism and required hormone replacement therapy with levothyroxine. Conclusions: Thyroid dysfunction secondary to immunotherapy is a common entity in our population. Clinical presentation is usually mild and does not require treatment discontinuation; however, due to the high incidence of these adverse events, non-oncology specialists must be familiar with the diagnosis and treatment of these alterations in order to provide multidisciplinary management.

KEY WORDS: Immune checkpoint inhibitors. Thyroiditis. Toxicity. Immunotherapy. Hypothyroidism

\section{Introducción}

El reciente conocimiento del papel que desempeña el sistema inmunológico en el control de la proliferación celular y el desarrollo de neoplasias ha revolucionado la oncología moderna. ${ }^{1}$ Con base en este conocimiento, en los últimos años se ha desarrollado un nuevo grupo de medicamentos oncológicos conocidos como inhibidores del punto de control inmunológico (IPCi). Los IPCi son anticuerpos monoclonales dirigidos contra pequeñas moléculas en la superficie de las células T. Estas moléculas, o puntos de control inmunológico, son el el antígeno-4 asociado con el linfocito T citotóxico (CTLA-4) y la molécula de muerte programada 1 (PD-1) y su ligando (PD-L1). Estas proteínas modulan la función de las células $T$ y desempeñan un papel crucial en la inmunidad antitumoral. ${ }^{2}$ En la actualidad existen seis IPCi para el tratamiento de tumores sólidos: ipilimumab (anticuerpo antiCTLA-4), nivolumab y pembrolizumab (con actividad anti-PD-1), así como atezolizumab, avelumab y durvalumab (que inhiben a PD-L1). ${ }^{3}$ Estos fármacos han aumentado la supervivencia de los pacientes con cáncer metastásico. ${ }^{4,5}$

$\mathrm{Al}$ inhibir estas moléculas, el tratamiento con IPCi produce una activación considerable del sistema inmune y con ello un incremento en su actividad antitumoral. Este mecanismo genera un estado de hiperinmunidad que altera los mecanismos de autotolerancia, lo cual puede producir eventos adversos autoinmunitarios en distintos órganos y sistemas. El sistema endocrino es particularmente susceptible a este fenómeno inmunológico $\mathrm{y}$, por ende, pueden existir deficiencias hormonales. La glándula tiroides es la afectada con mayor frecuencia, pudiéndose presentar hiper e hipotiroidismo. ${ }^{6}$ La hipófisis es la segunda glándula más afectada por estos fármacos, aunque en algunas series se refiere que incluso las alteraciones de esta glándula son más comunes que las de la tiroides. ${ }^{7,8}$ Con menos frecuencia se ha identificado diabetes mellitus y adrenalitis autoinmunitarias secundarias al uso de IPCi. ${ }^{9}$ En este estudio describimos nuestra experiencia en el diagnóstico y tratamiento de la disfunción tiroidea secundaria al tratamiento con IPCi.

\section{Métodos}

Se llevó a cabo un estudio retrospectivo en el cual se describieron las características clínicas, bioquímicas y de imagen de 122 pacientes que recibieron tratamiento con IPCi (ipilimumab, nivolumab y pembrolizumab) en un hospital privado de tercer nivel en la Ciudad de México, entre enero de 2014 y diciembre de 2017. Se compararon los pacientes con evidencia de disfunción tiroidea secundaria al tratamiento con $\mathrm{IPCi}$, contra los pacientes que no presentaron alteraciones hormonales posteriores al tratamiento. Los datos se obtuvieron del expediente médicos electrónico y el protocolo fue aprobado por el comité de bioética local, de acuerdo con los principios de la Declaración de Helsinki.

El tratamiento con IPCi fue administrado de acuerdo con las guías de práctica clínica, es decir, hasta que se observara progresión de la enfermedad o toxicidad severa que limitara su uso. ${ }^{10.11}$ Para clasificar a los pacientes que presentaron disfunción tiroidea se utilizó la Clasificación de Disfunción Tiroidea Secundaria a IPCi, publicada por el National Cancer Institute:

- Grado 1, cuadro asintomático que no requiere tratamiento específico.

- Grado 2, síntomas leves o moderados que limitan las actividades de la vida diaria.

- Grado 3, síntomas graves que requieren asistencia para actividades básicas de autocuidado.

- Grado 4, síntomas que ponen en riesgo la vida.

- Grado 5, enfermedad que implica la muerte debido a los efectos adversos. ${ }^{12}$

La medición de la hormona estimulante de la tiroides (TSH), T4 libre y T3 libre se realizó mediante inmunoensayos de electroquimioluminiscencia (Elecsys $₫$, Cobas 8000 , Roche, Basilea, Suiza). El inmunoensayo de TSH tiene un límite de detección de $0.005 \mu \mathrm{lU} / \mathrm{mL}$ y coeficientes de variación intra e interensayo $<1 \%$. El inmunoensayo de T4 libre tiene un límite de detección de $0.04 \mathrm{ng} / \mathrm{dL}$ y coeficientes de 
variación intra e interensayo $<10 \%$. El ensayo de T3 libre tiene un límite de detección de $0.4 \mathrm{pmol} / \mathrm{L}$, con un coeficiente de variación $<10 \%$.

Para el análisis estadístico, las variables numéricas se describen como media y desviación estándar o mediana y rangos intercuartílicos, dependiendo de la distribución de estas. Las variables categóricas se describen como porcentajes y frecuencias. Estimamos la prevalencia y la incidencia de tiroiditis secundaria a inmunoterapia por 1000 pacientes/mes. La comparación de las características clínicas y bioquímicas de los pacientes con y sin tiroiditis se realizó por medio de la prueba $U$ de Mann-Whitney o t de Student, de acuerdo con la distribución, en el caso de las variables continuas, y por medio de chi-cuadrada en las variables categóricas. Un valor de $p<0.05$ se consideró estadísticamente significativo. Para el análisis de las variables categóricas con múltiples comparaciones se utilizó la prueba de Kruksal-Wallis. Los datos fueron analizados con los programas estadísticos JMP 15 y SPSS versión 23.

\section{Resultados}

Durante el periodo de estudio, 122 pacientes con tumores sólidos en estadios avanzados fueron tratados con IPCi. La edad promedio fue de $61.95 \pm 13.4$ años, 40 (35.7\%) eran mujeres y 113 (92.6\%) tenían enfermedad en estadio clínico 4. Los tumores más comunes fueron cáncer de pulmón de células no pequeñas $(36.8 \%)$, melanoma $(29.5 \%)$ y carcinoma urotelial de vejiga (19.6\%). La mayoría de los pacientes (50\%) recibió pembrolizumab, al cual siguió el uso de nivolumab (33.6 \%) e ipilimumab (13.1\%); únicamente cuatro pacientes recibieron tratamiento combinado con ipilimumab y nivolumab. De los 122 pacientes, nueve $(7 \%)$ presentaron disfunción tiroidea secundaria al tratamiento. Aunque esto no fue estadísticamente significativo, los pacientes que presentaron disfunción tiroidea eran más jóvenes que quienes se mantuvieron eutiroideos (58.1 \pm 14.9 años versus $65.8 \pm 11.9$ años, $p=0.07$ ). La distribución de sexo femenino fue similar en ambos grupos (55.6\% versus $39.8 \%, p=0.35$ ), al igual que la proporción de pacientes con enfermedad en estadio clínico 4.

El cáncer de pulmón de células no pequeñas fue la neoplasia más común en los pacientes sin tiroiditis, mientras que el melanoma fue el tumor predominante en pacientes con disfunción tiroidea. La proporción de pacientes que recibieron cada tipo de IPCi fue similar en ambos grupos (Figura 1). Del grupo de

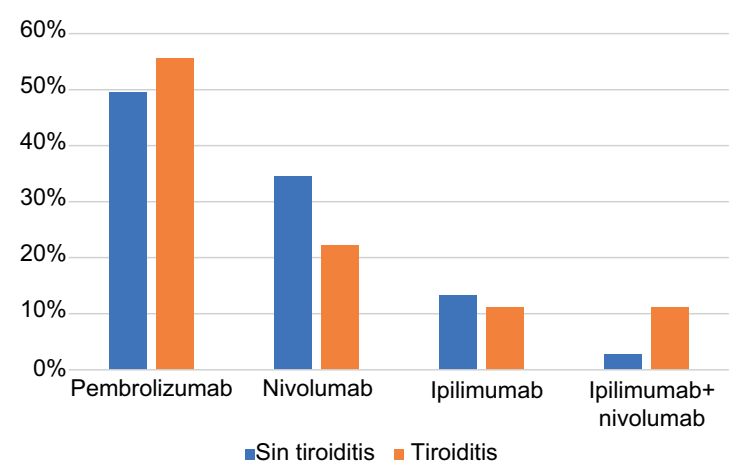

Figura 1. Pacientes con neoplasias malignas que presentaron o no tiroiditis secundaria a tratamiento por inhibidores de punto de control inmunológico $(n=122)$.

pacientes que desarrollaron tiroidopatía, cinco recibieron tratamiento con pembrolizumab, dos recibieron nivolumab y dos, ipilimumab (uno de ellos en combinación con nivolumab). En la Tabla 1 se comparan las características de los pacientes con disfunción tiroidea con las de los pacientes sin esta complicación.

Previo al inicio del tratamiento, todos los pacientes tenían pruebas de función tiroidea normales, con una mediana de TSH basal de 1.77 (0.3-4.2) $\mu \mathrm{IU} / \mathrm{mL}$. La disfunción tiroidea se presentó en una mediana de cuatro ciclos y 63 días después del inicio del tratamiento; sin embargo, ninguno de los pacientes requirió la suspensión del fármaco. De los nueve pacientes, solo uno se presentó inicialmente con tirotoxicosis, que posteriormente evolucionó a hipotiroidismo. La mediana de TSH al momento del diagnóstico fue de $22.58 \mu \mathrm{IU} / \mathrm{mL}$ (rango intercuartílico $[\mathrm{RIC}]=22.58$ 86.69), con un máximo de $233 \mu \mathrm{IU} / \mathrm{mL}$ y la mediana de T4L fue de $0.5 \mathrm{ng} / \mathrm{dL}(\mathrm{RIC}=0.4-0.9)$. La medición de anticuerpos antiperoxidasa tiroidea y antitiroglobulina se realizó en cuatro pacientes, de los cuales únicamente la mitad presentó resultados positivos. De los nueve pacientes, tres presentaron enfermedad leve (grado 1); tres, enfermedad moderada (grado 2) y tres, enfermedad grave (grado 3). Al término del seguimiento, la supervivencia en el grupo de pacientes con tiroiditis fue de $55 \%$, mientras que únicamente $38 \%$ de quienes no presentaron disfunción tiroidea sobrevivieron; sin embargo, esta relación no fue estadísticamente significativa. Ocho de los nueve pacientes recibieron tratamiento sustitutivo permanente con levotiroxina, con una mediana de seguimiento de tres meses $(\mathrm{RIC}=1.25-3.75)$ y una dosis de $112.5 \mu \mathrm{g}(\mathrm{RIC}=81-168)$. La prevalencia de tiroiditis durante el tiempo de estudio para esta población 
Tabla 1. Características basales de 122 pacientes que recibieron inmunoterapia de enero de 2014 a diciembre de 2017

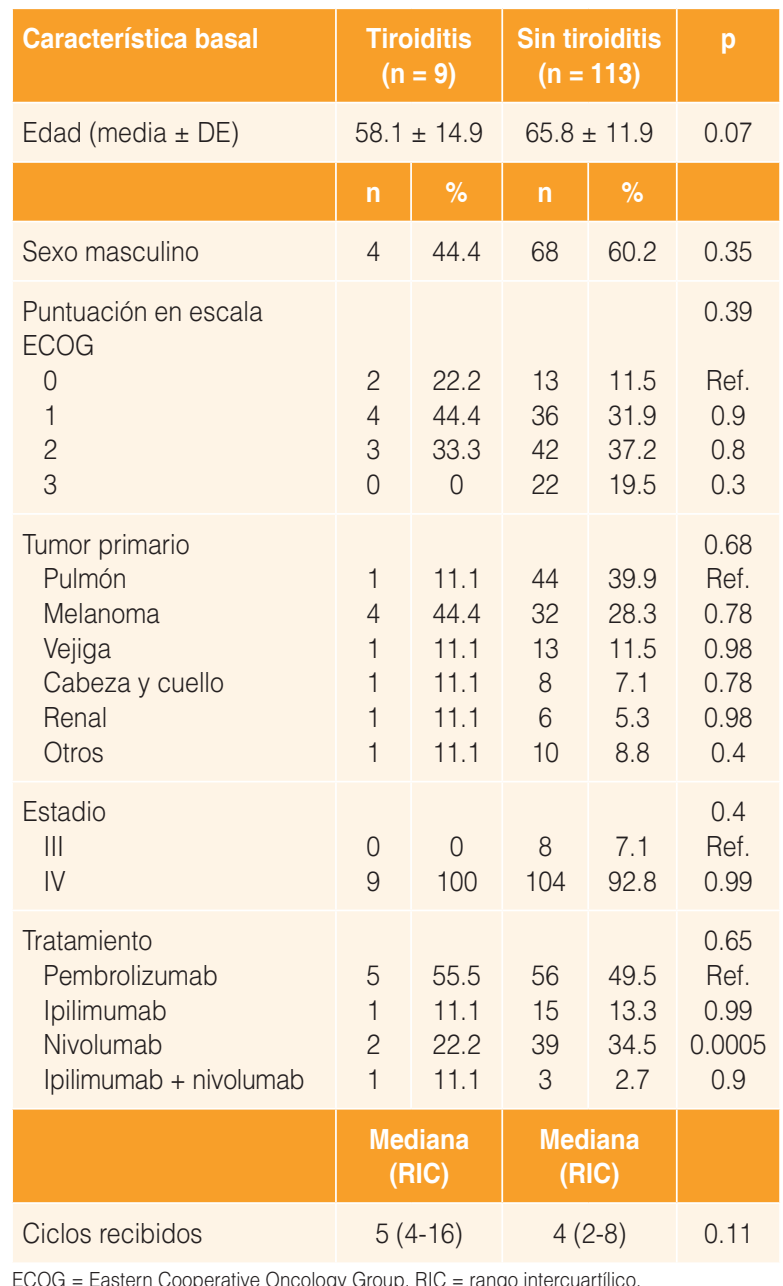

fue de $7 \%$, mientras que se estimó una incidencia de $21.4 \%$ pacientes/mes.

\section{Discusión}

Este es el primer estudio de tiroiditis secundaria al uso de inmunoterapia en población mexicana. Los IPCi representan una nueva esperanza para los pacientes con tumores metastáticos, ya que aumentan significativamente la supervivencia. ${ }^{13,14}$ Estos fármacos son anticuerpos monoclonales dirigidos contra moléculas que regulan la respuesta inmune y mantienen la homeostasis. Los principales blancos de estos anticuerpos son CTLA-4 (ipilumimab), PD-1 (nivolumab y pembrolizumab) y su ligando, PD-L1 (atezolizumab, avelumab y durvalumab)..$^{15} \mathrm{La}$ inhibición de estas moléculas lleva a una activación excesiva del sistema inmune, que si bien es útil para eliminar a las células tumorales, también causa disrupción de los mecanismos de autotolerancia, lo que lleva al desarrollo de fenómenos autoinmunes. ${ }^{16}$ Los órganos endocrinos, especialmente la glándula tiroides (pero también la hipófisis, el páncreas y las glándulas suprarrenales), son particularmente susceptibles a este fenómeno. ${ }^{17} \mathrm{El}$ uso de los IPCi, fármacos que han resultado útiles en el tratamiento de las neoplasias malignas, ha aumentado en los últimos años, por lo tanto, la presencia de la endocrinopatía asociada al tratamiento será un problema cada vez más común.

La incidencia de endocrinopatías autoinmunes asociadas al tratamiento con IPCi en pacientes con tumores sólidos es variable. La incidencia de hipofisitis se ha reportado en 9 a $17 \%$ de los pacientes tratados con ipilimumab, mientras que las tiroiditis son más frecuentes: se observan en 15 a $30 \%$ de los casos, dependiendo del IPCi utilizado. ${ }^{18}$ En una serie se reportó disfunción tiroidea en 29 de 126 pacientes (23\%) que recibieron ipilimumab en monoterapia, así como en 18 de 46 (39\%) pacientes en tratamiento con pembrolizumab o nivolumab. ${ }^{19}$ En otra serie, de 177 pacientes con melanoma metastásico, 24 (14\%) presentaron disfunción tiroidea. ${ }^{20}$ La prevalencia en nuestro estudio fue de $7 \%$ y la incidencia fue de $21.4 \%$ pacientes/mes, la cual es menor a la reportada previamente, sin embargo, nuestro estudio incluye pacientes con distintos tipos de tumores sólidos, no únicamente melanoma. Nuestro estudio muestra resultados similares a los de un metaanálisis reciente que incluyó 7551 pacientes y que reportó una incidencia de $6.6 \%$, con mayor riesgo en pacientes que recibieron tratamiento combinado con anti-CTLA-4 y anti-PD1. ${ }^{21}$

La fisiopatología de la tiroiditis relacionada con el tratamiento con IPCi es principalmente inflamatoria y destructiva, por lo que se presenta con una fase tirotóxica inicial secundaria a la liberación de hormonas tiroideas preformadas, aunque esta fase suele ser leve y normalmente pasa desapercibida. ${ }^{22}$ Posteriormente se presenta una fase de hipotiroidismo, que en algunos casos regresa al estado eutiroideo. ${ }^{23}$ En nuestra serie, ocho de los nueve pacientes se presentaron con hipotiroidismo con elevaciones variables de la TSH, desde una elevación mínima hasta $200 \mu \mathrm{UI} / \mathrm{mL}$. Únicamente un paciente se presentó con hipertiroidismo subclínico que no ameritó tratamiento médico y semanas después evolucionó a la fase de hipotiroidismo. Este hallazgo contrasta con datos de otras series, en las cuales la fase de 
hipertiroidismo se presentó en más de la mitad de los casos.

La presentación clínica y la severidad del cuadro es muy variable, en la mayoría de los casos con síntomas leves a moderados. ${ }^{24}$ Dos tercios de nuestros pacientes presentaron enfermedad grado 102 , mientras que solo en uno se observaron síntomas graves. Aunque no es una observación común, también se han reportado casos de enfermedad de Graves inducida por el tratamiento con IPCi. ${ }^{25}$ Similar a los hallazgos en estudios previos, los anticuerpos antitiroideos (antiperoxidasa tiroidea y antitiroglobulina) fueron positivos en la mitad de los pacientes en quienes se realizó la medición. ${ }^{26}$ De acuerdo con lo reportado en la literatura, la tiroiditis secundaria a los IPCi se diagnosticó en una mediana de tiempo de 63 días posterior al inicio del tratamiento. ${ }^{27}$ Durante el seguimiento, todos los pacientes se mantuvieron hipotiroideos y requirieron dosis estables de levotiroxina. En ningún paciente se documentó recuperación de la función tiroidea.

\section{Conclusiones}

Este es el primer análisis que evalúa la incidencia y las características clínicas de la tiroiditis relacionada con IPCi en América Latina, sin embargo, se trata de un estudio retrospectivo y con un número pequeño de casos, con las limitaciones que esto implica. La incidencia y el comportamiento clínico de la disfunción tiroidea en pacientes mexicanos que recibieron tratamiento con IPCi para tumores sólidos fueron similares a los reportados en series previas; sin embargo, la mayoría de los casos se presentó como hipotiroidismo. Debido a la naturaleza destructiva de esta enfermedad, la mayoría de los pacientes no regresó al estado eutiroideo y requirió tratamiento sustitutivo a largo plazo. Se deben solicitar pruebas de funcionamiento tiroideo antes de iniciar el tratamiento con IPCi y antes de cada ciclo, con el fin de diagnosticar y tratar a estos pacientes oportunamente y evitar cuadros severos y limitación en la calidad de vida. ${ }^{28}$

\section{Conflicto de intereses}

Los autores niegan conflictos de intereses.

\section{Financiamiento}

Ninguno.

\section{Responsabilidades éticas}

Protección de personas y animales. Los autores declaran que para esta investigación no se realizaron experimentos en seres humanos ni en animales.

Confidencialidad de los datos. Los autores declaran que en este artículo no aparecen datos de pacientes.

Derecho a la privacidad y consentimiento informado. Los autores declaran que en este artículo no aparecen datos de pacientes.

\section{Bibliografía}

1. Leach DR, Krummel MF, Allison JP. Enhancement of antitumor immunity by CTLA-4 blockade. Science. 1996;271:1734-136.

2. Byun DJ, Wolchok JD, Rosenberg LM, Girotra M. Cancer immunotherapy - immune checkpoint blockade and associated endocrinopathies. Nat Rev Endocrinol. 2017;13:195-207.

3. Brahmer JR, Lacchetti C, Schneider BJ, Atkins MB, Brassil KJ, Caterino JM, et al. Management of immune-related adverse events in patients treated with immune checkpoint inhibitor therapy: American Society of Clinical Oncology Clinical Practice Guideline. J Clin Oncol. 2017;36:1714-1768.

4. Dine J, Gordon R, Shames Y, Kasler MK, Barton-Burke M. Immune checkpoint inhibitors: an innovation in immunotherapy for the treatment and management of patients with cancer. Asia Pac J Oncol Nurs. 2017;4:127-135.

5. Hodi FS, O'Day SJ, McDermott DF, Weber RW, Sosman JA, Haanen JB, et al. Improved survival with ipilimumab in patients with metastatic melanoma. N Engl J Med. 2010;363:711-723.

6. Villa NM, Farahmand A, Du L, Yeh MW, Smooke-Praw S, Ribas A, et al. Endocrinopathies with use of cancer immunotherapies. Clin Endocrinol. 2018;88:327-332.

7. Di Dalmazi G, Ippolito S, Lupi I, Caturegli P. Hypophysitis induced by immune checkpoint inhibitors: a 10-year assessment. Expert Rev Endocrinol Metab. 2019;14:381-398.

8. Ferrari SM, Fallahi P, Galetta F, Citi E, Benvenga S, Antonelli A. Thyroid disorders induced by checkpoint inhibitors. Rev Endocr Metab Disord. 2018:19:325-333.

9. Castinetti F, Albarel F, Archambeaud F, Bertherat J, Bouillet B, Buffier P, et al. French Endocrine Society Guidance on endocrine side effects of immunotherapy. Endocr Relat Cancer. 2019;26:G1-G18.

10. Seth R, Messersmith H, Kaur V, Kirkwood JM, Kudchadkar R, McQuade JL, et al. Systemic therapy for melanoma: ASCO Guideline. J Clin Oncol. 2020;38:3947-3970.

11. Hanna N, Johnson D, Temin S, Baker S, Brahmer J, Elis PM, et al. Systemic therapy for stage IV non-small-cell lung cancer: American Society of Clinical Oncology Clinical Practice Guideline Update. J Clin Oncol. 2017:35:3484-3515.

12. Common Terminology Criteria for Adverse Events (CTCAE) version 5.0. EE. UU.: U.S. Department of Health and Human Services/National Institutes of Health/National Cancer Institute; 2017

13. Larkin J, Chiarion-Sileni V, Gonzalez R, Grob JJ, Cowey CL, Lao CD, et al. Combined nivolumab and ipilimumab or monotherapy in untreated melanoma. N Engl J Med. 2015;373:23-34.

14. Postow MA, Callahan MK, Wolchok JD. Immune checkpoint blockade in cancer therapy. J Clin Oncol. 2015;33:1974-1982.

15. La-Beck NM, Jean GW, Huynh C, Alzghari SK, Lowe DB. Immune checkpoint inhibitors: new insights and current place in cancer therapy. Pharmacotherapy. 2015;35:963-976

16. Buchbinder EI, Desai A. CTLA-4 and PD-1 pathways: similarities, differences, and implications of their inhibition. Am J Clin Oncol. 2016;39:98-106.

17. Ntali G, Kassi E, Alevizaki M. Endocrine sequelae of immune checkpoint inhibitors. Hormones (Athens). 2017;16:341-350.

18. Chang L, Barroso-Sousa, Tolaney, R, Hodi S, Kaiser U, Min L. Endocrine toxicity of cancer immunotherapy targeting immune checkpoints. Endocr Rev. 2019;40:17-65.

19. Morganstein D, Lai Z, Spain L, Diem S, Levine D, Mace C, et al. Thyroid abnormalities following the use of cytotoxic T-lymphocyte antigen-4 and programmed death receptor protein-1 inhibitors in the treatment of melanoma. Clin Endocrinol. 2017;86:614-620.

20. Scott ES, Long GV, Guminski A, Clifton-Bligh RJ, Menzies AM, Tsang VH The spectrum, incidence, kinetics and management of endocrinopathies with immune checkpoint inhibitors for metastatic melanoma. Eur $\mathrm{J}$ Endocrinol. 2018;178:175-182. 
21. Barroso-Sousa R, Barry WT, Garrido-Castro A, Pode, FS, Min L, Quero IE, et al. Incidence of endocrine dysfunction following the use of different immune checkpoint inhibitor regimens: a systematic review and meta-analysis. JAMA Oncol. 2018;4:173-182.

22. Lee H, Hodi FS, Giobbie-Hurder A, Ott PA, Buchbinder El, et al. Characterization of thyroid disorders in patients receiving immune checkpoint inhibition therapy. Cancer Immunol Res. 2017;5:1133-1140.

23. Mishima Y, Fukaishi T, Inase N, Isogai S. Nivolumab-induced hypophysitis, secondary adrenal insufficiency and destructive thyroiditis in a patient with lung adenocarcinoma. Intern Med. 2019;58:693-697.

24. Mace C, Diem S, Gore M, Larkin J, Morganstein D. Thyroid abnormalities during anti-PD1 cancer immunotherapy. Endocrine Abstracts. 2015:38:443.
25. Azmat U, Liebner D, Joehlin-Price A, Agrawal A, Nabhan F. Treatment of ipilimumab induced Graves' disease in a patient with metastatic melanoma. Case Rep Endocrinol. 2016;2016:2087525.

26. Varricchi G, Loffredo S, Marone G, Modestino L, Fallahi P, Ferrari SM, et al. The immune landscape of thyroid cancer in the context of immune checkpoint inhibition. Int J Mol Sci. 2019;20:3934.

27. Guaraí F, La Selva R, Xamã MT, D'Angelo V, Gorin D, Fava P, et al. Characterization and implications of thyroid dysfunction induced by immune checkpoint inhibitors in real-life clinical practice: a long-term prospective study from a referral institution. J Endocrinol Invest. 2018;41:549-556.

28. Spain L, Diem S, Larkin J. Management of toxicities of immune checkpoint inhibitors. Cancer Treat Rev. 2016;44:51-60. 